\title{
Protocolo Clínico para Control de Prótesis Fija Total con Carga Inmediata
}

\author{
Clinical Protocol for Control of an Immediately Loaded Implant-Supported Prosthesis
}

\author{
Emanuel Figueroa Ahumada \& lan Raby Olavarría
}

FIGUEROA, A. E. \& RABY, O. I. Protocolo clínico para control de prótesis fija total con carga inmediata. Int. J. Odontostomat. 12(3):296-303, 2018.

RESUMEN: Los protocolos odontológicos de controles son guías que tienen por objetivo optimizar la calidad de atención y estandarizar ciertos procedimientos para poder establecer mejores pronósticos. Varias complicaciones se pueden presentar posterior a la instalación de una prótesis fija total con carga inmediata (PFTC), desde el daño de la estructura protésica provisoria hasta la pérdida de la oseointegración de los implantes dentales. La escasa evidencia sobre el comportamiento clínico que se debe tener posterior a este tipo de tratamiento indica la necesidad de confección de un protocolo para poder mejorar las tasas de éxito. Para confeccionar el protocolo clínico se realizó una revisión sistemática de la literatura en la base de datos PubMed. Los filtros de búsqueda fueron ajustados de la siguiente manera. Se utilizaron los términos de búsqueda "immediate loading" AND "full arch" AND "Resonance frequency analysis". Los que fueron sometidos a los criterios de inclusión y exclusión, dando como resultado un total de 15 estudios. Todos los estudios seleccionados son ensayos clínicos controlados donde se encontraron complicaciones como fracturas, desalojos y desgaste de la estructura protésica, entre otros. En los resultados encontrados no existe evidencia sobre el número y rango de sesiones necesarias para el control clínico de una PFTCl. El momento más indicado para la extracción de la prótesis provisoria e instalación de la prótesis definitiva es durante el $3^{\circ}$ y $4^{\circ}$ mes posterior a la carga inmediata. Existe escasa evidencia sobre el número, momento y tipo de examen imageneológico a solicitar. Por lo que este protocolo clínico será una guía para los controles que se realicen posterior a una instalación de una PFTCI.

PALABRAS CLAVE: carga inmediata, arco completo, análisis de frecuencia de resonancia.

\section{INTRODUCCIÓN}

Esposito en su última revisión bibliográfica (Esposito et al., 2009) sobre protocolos de carga, concluyó que la carga inmediata es un procedimiento que se puede efectuar con un éxito predecible, pero la tendencia indica que los implantes con carga inmediata fallan con más frecuencia que los con carga convencional. Además, se concluyó que la carga inmediata en maxilar inferior está bien documentada, no así en maxilar superior. La literatura sobre carga inmediata con prótesis fija total en maxilar superior muestra resultados exitosos si se sabe utilizar los criterios de selección de pacientes, implantes, tratamiento quirúrgico y protésico.

Los estudios de pacientes rehabilitados con $\mathrm{PFTCl}$ mencionan que las complicaciones, tanto biológicas como protésicas son muy escasas
(Peñarrocha-Oltra et al., 2014). Por lo general las complicaciones intraoperatoria son raras, y en el caso de las postoperatorias inmediata las únicas son la hinchazón y el dolor, siendo estas de poca gravedad (Degidi et al., 2005; Kinsel et al., 2007; Weber et al., 2009). Por otra parte, un estudio de Van Steenberghe aclara que durante el proceso de oseointegración se podría producir fractura de la estructura protésica provisoria en una carga inmediata (Van Steenberghe et al., 2005), presentándose de forma frecuente y con un grado de complejidad bajo al momento de querer reparar la estructura (Pieri et al., 2009). Una vez concretada la oseointegración se pueden encontrar complicaciones biológicas, como lo son la perimplantitis y la mucositis (Van Steenberghe et al., 2005). Otros estudios, enfatizan que las complicaciones más graves que conducen al fracaso de los implantes es la fractu- 
ra total de la prótesis acrílica producto de fuerzas nocivas (Grunder, 2001; Malo et al., 2005).

Al evaluar la literatura, resalta el hecho de que los pocos estudios en PFTCl disponibles usan diferentes procedimientos quirúrgicos, tipos de prótesis, tiempos de carga y diferentes diseños de investigación (Peñarrocha-Oltra et al., 2014). No obstante, aunque la evidencia científica en $\mathrm{PFTCI}$ sea poco concreta y estandarizada, esta técnica es muy utilizada por los clínicos, puesto que tiene una tasa de éxito y sobrevida del implante muy similar a la del protocolo convencional (Horwitz et al., 2007; Sanz et al., 2013).

Teniendo en cuenta lo anterior, y el hecho de que no existen estudios sobre el control clínico en PFTCl, es que nace la necesidad de confeccionar un protocolo clínico que guíe al profesional, para así poder efectuar un análisis y control más acabado de alguna eventualidad o complicación posterior a una carga inmediata, y de esta manera otorgar un mayor confort de tratamiento al paciente.

\section{MATERIAL Y MÉTODO}

En esta revisión bibliográfica de tipo narrativa, se realizó una búsqueda electrónica el 3 de marzo de 2016 en la base de datos PubMed. Para poder efectuar una investigación acabada los filtros de búsqueda fueron ajustados de la siguiente manera.

1.Tipo de artículo: Ensayo clínico, revisión sistémica y narrativa.

2. Disponibilidad del texto: Texto completo, texto completo gratis y abstracto.

3. Años de publicación: Desde el año 2000 en adelante.

4.Especie: Humanos.

5. Idiomas: Inglés y español.

Una vez aplicados los filtros, se procedió a ingresar los términos de búsqueda: "immediate loading" AND "full arch" AND "Resonance frequency analysis", de forma individual y combinadas. Este procedimiento dio como resultado un total de 106 investigaciones.

Posterior a esto se procedió a aplicar los criterios de inclusión:

1. Carga inmediata en prótesis fija total para maxilar superior, inferior o ambas.
2. Seguimiento de a los menos 6 meses posterior a la carga inmediata.

3. Estudios que mencionen número y rango de sesiones necesarias para el control clínico de la carga inmediata.

4. Estudios que mencionen tipo, número y rango de exámenes imagenológico a solicitar.

5. Estudios que mencionen el momento en que se debe retirar por primera vez la prótesis provisoria.

6 . Estudios relacionados con la evaluación de la cicatrización ósea posterior a una carga inmediata.

7. Estudios que mencionen tipos de complicaciones en prótesis fija total con carga inmediata.

Lo anterior arrojó un total de 19 estudios, a los cuales se les aplicaron los criterios de exclusión:

1. Paciente fumadores pesados (más de 10 cigarrillos diarios).

2. Pacientes sistémicamente comprometidos.

3. Paciente con enfermedad periodontal activa.

4. Implantes cigomáticos.

5. Implantes cortos.

6. Implantes de zirconio.

7. Cirugía guiada por computador.

8. CAD-CAM.

9. Torque de inserción del implante menor a 35 newton.

Producto de lo anterior, este proceso arrojo un total de 15 estudios que cumplían con los criterios de selección.

\section{RESULTADOS}

Los resultados vistos en los ECR (ensayos clínicos randomizados) que utilizaron protocolo de carga inmediata con prótesis fija de arco completo varían tanto en el número, tiempo y periodicidad en que citaron a control a cada uno de los pacientes. Podemos ver que en algunas investigaciones, independiente de su objetivo, el primer control fue a la $1^{\circ}$ semana después de la carga inmediata, en otros a los 10 días $\mathrm{o}$ incluso entre el $1^{\circ}$ y $2^{\circ}$ mes (Tabla I).

Sobre el retiro de la prótesis provisoria por primera vez, como también la instalación de la prótesis definitiva, se observó que los estudios muestran diferencias en el momento que este procedimiento se lleva a cabo, e incluso en algunos no informan este hecho. El retiro del provisorio va desde los 10 días 
Tabla I. Estudios con PFTCI y sus diferencias en número, momento y frecuencia de citación a controles. Ausencia de control o información no especificada en la investigación.

\begin{tabular}{|c|c|c|c|c|c|c|c|}
\hline Autor & Año & $\begin{array}{l}\text { Tipo de } \\
\text { estudio }\end{array}$ & Análisis & $1^{\circ}$ control & $2^{\circ}$ control & $3^{\circ}$ control & Otros \\
\hline Bergkvist et al. & 2009 & ECC & $\begin{array}{l}\text { Sobrevida de la carga } \\
\text { inmediata. }\end{array}$ & 1 mes & 8 meses & 20 meses & 32 meses \\
\hline Agliardi et al. & 2010 & ECC & $\begin{array}{l}\text { Sobrevida de la carga } \\
\text { inmediata. }\end{array}$ & 1 semana & 3 meses & 6 meses & 9 meses \\
\hline Acocella et al. & 2012 & ECC & $\begin{array}{l}\text { Efectividad de la carga } \\
\text { inmediata. }\end{array}$ & 10 días & 6 meses & 12 meses & - \\
\hline Ganeles et al. & 2001 & ECC & $\begin{array}{l}\text { Efectividad de la carga } \\
\text { inmediata. }\end{array}$ & 1 semana & 2 semanas & 3 semanas & $1 \mathrm{vez}$ al mes \\
\hline Maló et al. & 2012 & ECC & $\begin{array}{l}\text { Sobrevida de la carga } \\
\text { inmediata. }\end{array}$ & $1 \mathrm{mes}$ & 2 meses & 3 meses & $4-6-7-9-10-11-12$ \\
\hline Piano et al. & 2016 & ECC & $\begin{array}{l}\text { Efectividad de la carga } \\
\text { inmediata con }\end{array}$ & 1 semana & 2 semanas & 3 meses & 12 meses \\
\hline Testori et al. & 2008 & ECC & $\begin{array}{l}\text { Efectividad de la carga } \\
\text { inmediata con }\end{array}$ & 1 mes & 3 meses & 6 meses & 12 meses \\
\hline Eccellente et al. & 2011 & ECC & $\begin{array}{l}\text { Efectividad dë la ćarga } \\
\text { inmediata. }\end{array}$ & 2 semanas & 8 semanas & 10 semanas & - \\
\hline
\end{tabular}

PTFCl: Prótesis fija total con carga inmediata.

hasta los 6 meses. La instalación de prótesis definitiva va desde los 3 a 6 meses. En algunos casos algunos estudios no especifican esta información (Tabla II).

En la toma de exámenes imageneológicos, los resultados arrojaron diferencias en el tipo de examen a solicitar, momento y número. La primera toma va desde inmediatamente después de la carga inmediata hasta 32 meses después. Esto último está muy relacionado con el tiempo de duración que tenía destinado el estudio. Se puede observar que los exámenes imageneológicos más solicitados eran dos: radiografías periapicales y panorámicas. (Tabla III).

Al momento de observar la presencia o ausencia de complicaciones protésicas en una PFTCl, la literatura no es muy específica en demostrar estadísticamente este fenómeno. No obstante, varios estudios en que se manifestó la fractura completa de la estructura protésica, concuerdan que esta es la complicación más grave (Tabla IV).

En otro estudio (Drago, 2017), 128 pacientes fueron sometidos a PFTCI, donde se dejó como provisorio una prótesis acrílica atornillada de arco completo. Las complicaciones de la prótesis provisoria se dividieron casi por igual en maxilar (19) y mandíbula (18). Maxilar superior tuvo un poco más de fracturas protésicas (15) que en mandíbula (11) (Tabla V).

De todos los estudios analizados, solo uno fue específico en la resolución de las complicaciones que se pudiesen presentar en una prótesis provisoria de arco completo posterior a la carga inmediata. (Malo et al., 2011) (Tabla VI).

Tabla II. Estudios con PFTCI y sus diferencias en el momento del retiro de la prótesis provisoria e instalación de la estructura definitiva.

\begin{tabular}{|c|c|c|c|c|c|}
\hline Autor & Año & $\begin{array}{l}\text { Tipo de } \\
\text { estudio }\end{array}$ & Análisis & $\begin{array}{l}\text { Retiro de prótesis } \\
\text { provisoria }\end{array}$ & $\begin{array}{c}\text { Instalación Prótesis } \\
\text { definitiva }\end{array}$ \\
\hline Bergkvist et al. & 2009 & ECC & Sobrevida de la carga inmediata. & 10 a 12 días & 15 semanas \\
\hline Agliardi et al. & 2010 & $\mathrm{ECC}$ & Sobrevida de la carga inmediata. & 4 a 6 meses & 4 a 6 meses \\
\hline Acocella et al. & 2012 & ECC & Efectividad de la carga inmediata. & 3 meses & - \\
\hline Ganeles et al. & 2001 & $\mathrm{ECC}$ & Efectividad de la carga inmediata. & - & 3 a 6 meses \\
\hline Maló et al. & 2012 & $\mathrm{ECC}$ & Sobrevida de la carga inmediata. & - & 6 meses \\
\hline Piano et al. & 2016 & $\mathrm{ECC}$ & $\begin{array}{l}\text { Efectividad de la carga inmediata con } \\
\text { implantes inclinados. }\end{array}$ & 3 meses & - \\
\hline Testori et al. & 2008 & ECC & $\begin{array}{l}\text { Efectividad de la carga inmediata con } \\
\text { implantes inclinados. }\end{array}$ & - & 3 meses \\
\hline Eccellente et al. & 2011 & ECC & Efectividad de la carga inmediata. & 2 semanas & 6 meses \\
\hline
\end{tabular}

Información no especificada en la investigación. PTFCl: Prótesis fija total con carga inmediata. 
Tabla III. Estudios con PFTCl y sus diferencias en el número, momento y tipo de examen imagenológico a solicitar.

\begin{tabular}{|c|c|c|c|c|c|c|c|}
\hline Autor & Año & $\begin{array}{l}\text { Tipo de } \\
\text { estudio }\end{array}$ & Análisis & $1^{\circ}$ Toma & $2^{\circ}$ Toma & $3^{\circ}$ Toma & Tipo \\
\hline Bergkvist et al. & 2009 & ECC & $\begin{array}{l}\text { Sobrevida de la } \\
\text { carga inmediata. }\end{array}$ & $\begin{array}{l}\text { Inmediatamente posterior } \\
\text { a la carga inmediata. }\end{array}$ & 8 meses & $\begin{array}{l}20 \text { y } 32 \\
\text { meses }\end{array}$ & Periapical \\
\hline Agliardi et al. & 2010 & ECC & $\begin{array}{l}\text { Sobrevida de la } \\
\text { carga inmediata. }\end{array}$ & 6 meses & 12 meses & - & $\begin{array}{l}\text { Panorámica } \\
\text { y periapical }\end{array}$ \\
\hline Eccellente et al. & 2011 & ECC & $\begin{array}{l}\text { Efectividad de la } \\
\text { carga inmediata. }\end{array}$ & 6 meses & 12 meses & - & Panorámica \\
\hline Ganeles et al. & 2001 & ECC & $\begin{array}{l}\text { Efectividad de la } \\
\text { carga inmediata. }\end{array}$ & 3 meses & 6 meses & - & $\begin{array}{l}\text { Panorámica } \\
\text { y periapical }\end{array}$ \\
\hline Maló et al. & 2012 & ECC & $\begin{array}{l}\text { Sobrevida de la } \\
\text { carga inmediata. }\end{array}$ & 6 meses & 12 meses & - & $\begin{array}{l}\text { Panorámica } \\
\text { y periapical }\end{array}$ \\
\hline Piano et al. & 2016 & ECC & $\begin{array}{l}\text { Efectividad de la } \\
\text { carga inmediata con } \\
\text { implantes inclinados. }\end{array}$ & $\begin{array}{l}\text { Inmediatamente posterior } \\
\text { a la carga inmediata. }\end{array}$ & 12 meses & - & Periapical \\
\hline Testori et al. & 2008 & ECC & $\begin{array}{l}\text { Efectividad de la } \\
\text { carga inmediata con } \\
\text { implantes inclinados. }\end{array}$ & 1 mes & 3 meses & 6 meses & Periapical \\
\hline Acocella et al. & 2012 & ECC & $\begin{array}{l}\text { Efectividad de la } \\
\text { carga inmediata. }\end{array}$ & - & - & - & - \\
\hline
\end{tabular}

Ausencia de toma de radiografía o información no especificada en la investigación. PTFCl: Prótesis fija total con carga inmediata.

\section{Protocolo para el control clínico de prótesis fija total con carga inmediata.}

\section{$1^{\circ}$ Semana:}

1. Torque manual a tornillos protésicos más cierre temporal de chimenea (Bergkvist et al., 2009).

2.Toma de set de periapical.

a. Registrar medición del estado de hueso perimplantar por implante.

Se debe medir desde unión implante-pilar hasta hueso marginal en la unión hueso-implante. Primero por mesial y luego por distal en relación al implante. Se suman ambas mediciones y se dividen por dos. Estamedición se debe efectuar por cada implante y registrar.

3. Ajuste de oclusión:

a. Se debe disminuir la altura cúspidea e idealmente aplanar cúspides guía.

b. La guía anterior en protrusión y lateralidad debe ser lo más plana posible.

c. La prótesis provisoria en su zona posterior deben tener una mesa oclusiva estrecha en comparación con la dentición natural.

d. Los contactos oclusales deben ser axiales al implante.

e. Es importante una distribución simétrica de los con- tactos oclusales, especialmente en el inicio de cicatrización de los tejidos (Drago et al., 2011).

f. Fuerzas parafuncionales pueden interrumpir el periodo de oseointegración.

g. Se debe evitar el cantiléver para que no existan fuerzas no axiales (Kinsel \& Liss, 2005).

4. Indicaciones para el paciente:

a. Dieta blanda por 3 meses.

b. Enjuagues suaves de $15 \mathrm{ml}$ de clorhexidina al $0,12 \%, 2$ veces al día por 3 semanas (Ganeles et al., 2001).

c. Inmediatamente después de instalar la estructura provisoria el cepillado solo se debe restringir a la superficie de la prótesis (Corbella et al., 2011).

d. Luego de dos semanas se debe utilizar un cepillo interdental pequeño y de cerdas suaves. Además una seda dental con esponja. El barrido mecánico se debe limitar solo a los cuellos de los implantes y la superficie apical de la prótesis (Corbella et al.).

e. Posterior a las dos semanas se debe indicar un cepillo interdental más grueso, debido al aumento del tamaño del espacio entre la prótesis y el tejido perimplantario. Esto por el proceso de cicatrización (Corbella et al.).

\section{$2^{\circ}$ y $3^{\circ}$ semanas:}

1. Chequear estado de tornillos. Torque manual y cierre temporal de chimeneas (Bergkvist et al.). 


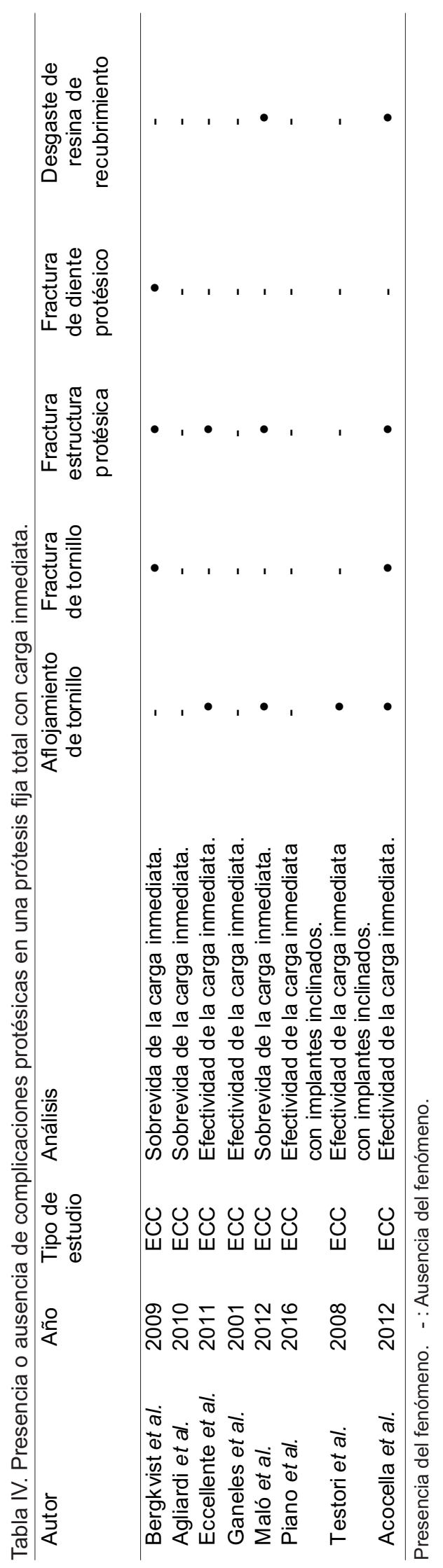

2. Ajuste de oclusión.

3. Reforzar y revaluar indicaciones del paciente dadas en la primera semana.

4. Evaluar posibles complicaciones (Tabla VI) (Maló, 2011).

\section{Complicaciones y su resolución}

\section{$4^{\circ}$ semana:}

1.Chequear estado de tornillo. Torque de tornillo protésico y cierre de chimenea de forma definitiva (Bergkvist et al.).

2. Ajuste de oclusión.

3. Reforzar y revaluar indicaciones del paciente dadas en la primera semana.

4. Evaluar posibles complicaciones (Tabla VI) (Maló, 2011).

\section{$2^{\circ}$ mes:}

1. Chequear estado del cierre de chimenea.

2. Ajuste de oclusión.

3. Reforzar y revaluar indicaciones del paciente dadas en la primera semana.

4. Evaluar posibles complicaciones. (Tabla VI)(Maló et al., 2011).

\section{$3^{\circ}$ a $4^{\circ}$ mes:}

1. Set de radiografías periapical. Registro de mediciones al igual que en primera semana y comparar cambios en margen óseo perimplantar.

2. Retiro de prótesis provisoria (Concejo Cútoli \& Montesdeoca García, 2005).

3. Higienizar implantes dentales.

4. Instalación de prótesis definitiva con tornillos nuevos. En un principio cerrar chimenea y torquear tornillos de forma provisoria. Controlar y evaluar cierre de chimenea y torque de tornillos protésicos de forma definitiva.

5. Control de oclusión.

6. Indicaciones para el paciente:

a. Al momento de cambiar la estructura protésica provisoria por una definitiva se debe indicar nuevamente un cepillo máspequeño, puesto que el contacto de una prótesis definitiva comparada con la de un provisorio es mucho más íntimo (Corbella et al.).

b. Indicar waterpik y seda dental super-floss.

\section{$6^{\circ}$ mes:}

1. Toma de radiografía panorámica. Evaluar integridad de estructura protésica y su relación con implantes y tejido colindante.

2. Ajuste de oclusión.

3. Reforzar y re-evaluar técnica de higiene.

4. A estas alturas los implantes ya están oseointegrados, por lo tanto se debe evaluar el perfil de riesgo del paciente para establecer la frecuencia de las mantenciones. 
Tabla V. Presencia del tipo de fractura en prótesis provisoria en una PFTCl.

\begin{tabular}{lcccc}
\hline Prótesis provisoria & Total & $\begin{array}{c}\text { Fractura } \\
\text { Estructura protésica }\end{array}$ & $\begin{array}{c}\text { Fractura dentaria } \\
\text { adhesiva }\end{array}$ & $\begin{array}{c}\text { Fractura dentaria } \\
\text { cohesiva }\end{array}$ \\
\hline Maxilar & 19 & 15 & 0 & 4 \\
Mandibula & 18 & 11 & 0 & 7 \\
Total & 37 & 26 & 0 & 11 \\
\hline
\end{tabular}

PTFCI: Prótesis fija total con carga inmediata

Tabla VI: Complicaciones protésicas y su resolución para PFTCI.

\begin{tabular}{lll}
\hline \multicolumn{1}{c}{ Complicación } & \multicolumn{1}{c}{ Resolución } \\
\hline Fractura completa de la estructura protésica. & 1. Reparación de prótesis. \\
& 2. Ajuste de la oclusión. \\
& 3. Plano de relajación. \\
Aflojamiento del tornillo protésico. & 4. Recomendar al paciente de no sobrecargar la prótesis. \\
& 1. Apretar tornillo. \\
Desgaste del material de la prótesis y del tornillo & 2. Ajuste de la oclusión. \\
protésico. & 1. Reemplazo del tornillo protésico. \\
& 2. Control de oclusión. \\
\hline
\end{tabular}

PFTCl: Prótesis fija total con carga inmediata.

\section{CONCLUSIONES}

No existe evidencia científica que determine cuales son los procedimientos clínicos post-operatorios para el control quirúrgico y protésicos en pacientes rehabilitados mediante prótesis fija total con carga inmediata, ya que en la literatura no hay estudios relacionados sobre el tema.

La presencia de complicaciones posterior a la instalación de una prótesis fija total con carga inmediata hace necesaria la confección de este protocolo de control clínico para asegurar un tratamiento exitoso y benéfico para el paciente. La complicación más grave es la fractura total de la estructura protésica y la causa más común es la sobrecarga oclusal.

No existe evidencia concreta sobre el número, momento y frecuencia de sesiones necesarias para el control clínico de una PFTCI. No obstante, es aconsejable llevar un control minucioso entre el día de la carga inmediata y el $4^{\circ}$ mes. Esto debido al intenso proceso de remodelación ósea.

El momento más indicado para la extracción de la prótesis provisoria por primera vez e instalación de la prótesis fija total definitiva es durante el $3^{\circ}$ y $4^{\circ}$ mes posterior a la carga inmediata. Puesto a que en ese momento la estabilidad del implante ya es segura.
La evidencia científica sobre el número, momento y tipo de examen imageneológico a solicitar es escasa e inconsistente. Sin embargo, solicitar un set de radiografías periapical el día de la carga y 3 meses después es muy ventajoso. Esto se debe a que entrega información relacionada con la integridad de las estructuras protésicas junto con la implantaría el día de la carga, y como el intenso remodelado ósea se desenvuelve alrededor de los implantes.

Es aconsejable llevar a cabo nuevas investigaciones que aporten de forma objetiva y concreta conocimiento sobre el correcto control clínico de prótesis fijas totales con carga inmediata.

FIGUEROA, A. E. \& RABY, O. I. Clinical protocol for control of an immediately loaded implant-supported prosthesis. Int. J. Odontostomat., 12(3):296-303, 2018.

ABSTRACT: Dental protocols are guidelines used to optimize service quality and to standardize procedures to establish a better prognosis. There are many complications that can occur after installation of a an immediately loaded implant-supported prosthesis for rehabilitation. These may generate damage to the provisional structure and even loss of osseointegration from the dental implants. Limited evidence on the clinical approach required to monitor these procedures, 
makes it necessary to establish clinical protocols for treatment control, and increases the success rate. In order to develop such protocols a systematic research was carried out in the Pubmed database. The filters used were "immediate loading", "full arch" and "Resonance frequency analysis". The results were subject to the inclusion and exclusion criteria. A total of 15 studies were selected. All of the studies were randomized clinical trials. An important number of complications were recorded, such as fractures, dislodgment and wear of the prosthesis structure. There is no evidence about the number and range of sessions needed to assess the clinical control of an immediate loading fixed rehabilitation. The best time to remove the provisional prosthesis and install the restorative rehabilitation is during the third and fourth month following immediate loading. Limited evidence was found regarding the number, time and type of radiological images requested for clinical support. Therefore, the clinical protocol developed by the authors will be a guide for future controls related to an immediately loaded implant-supported prosthesis.

KEY WORDS: immediate loading, full arch, Resonance frequency analysis.

\section{REFERENCIAS BIBLIOGRÁFICAS}

Acocella, A.; Ercoli, C.; Geminiani, A.; Feng, C.; Billi, M.; Acocella, G.; Giannini, D. \& Sacco, R. Clinical evaluation of immediate loading of electroeroded screw-retained titanium fixed prostheses supported by tilted implant: a multicenter retrospective study. Clin. Implant Dent. Relat. Res., 14 Suppl. 1:e98-108, 2012

Agliardi, E.; Clericò, M.; Ciancio, P. \& Massironi, D. Immediate loading of full-arch fixed prostheses supported by axial and tilted implants for the treatment of edentulous atrophic mandibles. Quintessence Int., 41(4):285-93, 2010.

Bergkvist, G.; Nilner, K.; Sahlholm, S.; Karlsson, U. \& Lindh, C. Immediate loading of implants in the edentulous maxilla: use of an interim fixed prosthesis followed by a permanent fixed prosthesis: a 32-month prospective radiological and clinical study. Clin. Implant Dent. Relat. Res., 11(1):1-10, 2009.

Concejo Cútoli, C. \& Montesdeoca García, N. Carga inmediata en implantes dentales. Rev. Esp. Cir. Oral Maxilofac., 27(5):25569,2005

Corbella, S.; Del Fabbro, M.; Taschieri, S.; De Siena, F. \& Francetti, L. Clinical evaluation of an implant maintenance protocol for the prevention of peri-implant diseases in patients treated with immediately loaded full-arch rehabilitations. Int. J. Dent. Hyg., 9(3):216-22, 2011.

Degidi, M.; Piattelli, A.; Felice, P. \& Carinci, F. Immediate functional loading of edentulous maxilla: a 5-year retrospective study of 388 titanium implants. J. Periodontol., 76(6):1016-24, 2005.

Drago, C. Cantilever lengths and anterior-posterior spreads of interim, acrylic resin, full-arch screw-retained prostheses and their relationship to prosthetic complications. J. Prosthodont., 26(6):502-507, 2017.

Drago, C.; del Castillo, R. \& Peterson, T. Immediate occlusal loading in edentulous jaws, CT-guided surgery and fixed provisional prosthesis: a maxillary arch clinical report. J. Prosthodont., 20(3):209-17, 2011.
Duvina, M.; Barbato, L.; Buti J.; Delle Rose, G.; Brancato, L.; Casella, G.; Longoni, S.; Sartori, M.; Amunni, F. \& Tonelli, P. Immediate loading with LASER-treated surface implant: twoyear resonance frequency analysis follow-up. J. Craniofac. Surg., 24(3):849-55, 2013.

Eccellente, T.; Piombino, M.; Piattelli, A.; D'Alimonte, E.; Perrotti, V. \& lezzi, G. Immediate loading of dental implants in the edentulous maxilla. Quintessence Int., 42(4):281-9, 2011.

Erkapers, M.; Ekstrand, K.; Baer, R. A.; Toljanic, J. \& Thor, A. Patient satisfaction following dental implant treatment with immediate loading in the edentulous atrophic maxilla. Int. J. Oral Maxillofac. Implants, 26(2):356-64, 2011.

Esposito, M.; Grusovin, M. G.; Maghaireh, H. \& Worthington, H. Interventions for replacing missing teeth: different times for loading dental implants. Cochrane Database Syst. Rev., (3):CD003878, 2013

Esposito, M.; Grusovin, M. G.; Willings, M.; Coulthard, P. \& Worthington, $\mathrm{H}$. V. The effectiveness of immediate, early, and conventional loading of dental implants: a Cochrane systematic review of randomized controlled clinical trials. Int. J. Oral Maxillofac. Implants, 22(6):893-904, 2007.

Ganeles, J.; Rosenberg, M. M.; Holt, R. L. \& Reichman, L. H. Immediate loading of implants with fixed restorations in the completely edentulous mandible: report of 27 patients from a private practice. Int. J. Oral Maxillofac. Implants, 16(3):41826, 2001.

Grunder, U. Immediate functional loading of immediate implants in edentulous arches: two-year results. Int. J. Periodontics Restorative Dent., 21(6):545-51, 2001.

Horwitz, J.; Zuabi, O.; Peled, M. \& Machtei, E. E. Immediate and delayed restoration of dental implants in periodontally susceptible patients: 1-year results. Int. J. Oral Maxillofac. Implants, 22(3):423-9, 2007.

Ibañez, J. C.; Tahhan, M. J.; Zamar, J. A.; Menendez, A. B.; Juaneda, A. M.; Zamar, N. J. \& Monqaut, J. L. Immediate occlusal loading of double acid-etched surface titanium implants in 41 consecutive full-arch cases in the mandible and maxilla: 6- to 74-month results. J. Periodontol., 76(11):1972-81, 2005.

Kinsel, R. P. \& Liss, M. Retrospective analysis of 56 edentulous dental arches restored with 344 single-stage implants using an immediate loading fixed provisional protocol: statistical predictors of implant failure. Int. J. Oral Maxillofac. Implants, 22(5):823-30, 2007.

Maló, P.; de Araújo Nobre, M.; Lopes, A.; Francischone, C. \& Rigolizzo, M. "All-on-4" immediate-function concept for completely edentulous maxillae: a clinical report on the medium (3 years) and long-term (5 years) outcomes. Clin. Implant Dent. Relat. Res., 14 Suppl. 1:e139-50, 2012.

Peñarrocha-Oltra, D.; Covani, U.; Peñarrocha-Diago, M. \& Peñarrocha-Diago, M. Immediate loading with fixed full-arch prostheses in the maxilla: review of the literature. Med. Oral Patol. Oral Cir. Bucal, 19(5):e512-7, 2014.

Piano, S.; Romeo, E.; Sbricoli, L.; Pisoni, G.; Cea, N. \& Lops, D. Simplified procedure for the immediate loading of a complete fixed prosthesis supported by four implants in the maxillary jaw: a 2-year prospective study. Clin. Oral Implants Res., 27(12):e154-60, 2016.

Pieri, F.; Aldini, N. N.; Fini, M. \& Corinaldesi, G. Immediate occlusal loading of immediately placed implants supporting fixed restorations in completely edentulous arches: a 1-year prospective pilot study. J. Periodontol., 80(3):411-21, 2009.

Sanz, M.; Ivanoff, C. J.; Weingart, D.; Wiltfang, J.; Gahlert, M.; Cordaro, L.; Ganeles, J.; Bragger, U.; Jackowski, J.; Martin, W. C.; Jung, R. E.; Chen, S. \& Hammerle, C. Clinical and radiologic outcomes after submerged and transmucosal implant placement with two-piece implants in the anterior maxilla and 
mandible: 3-year results of a randomized controlled clinical trial. Clin. Implant Dent. Relat. Res., 17(2):234-46, 2015.

Testori, T.; Del Fabbro, M.; Capelli, M.; Zuffetti, F.; Francetti, L. \& Weinstein, R. L. Immediate occlusal loading and tilted implants for the rehabilitation of the atrophic edentulous maxilla: 1-year interim results of a multicenter prospective study. Clin. Oral Implants Res., 19(3):227-32, 2008.

van Steenberghe, D.; Glauser, R.; Blombäck, U.; Andersson, M.; Schutyser, F.; Pettersson, A. \& Wendelhag, I. A computed tomographic scan-derived customized surgical template and fixed prosthesis for flapless surgery and immediate loading of implants in fully edentulous maxillae: a prospective multicenter study. Clin. Implant Dent. Relat. Res., 7 Suppl. 1:S111-20, 2005.

Weber, H. P.; Morton, D.; Gallucci, G. O.; Roccuzzo, M.; Cordaro, L. \& Grutter, L. Consensus statements and recommended clinical procedures regarding loading protocols. Int. J. Oral Maxillofac. Implants, 24 Suppl.:180-3, 2009.

Woda, A.; Nicolas, E.; Mishellany-Dutour, A.; Hennequin, M.; Mazille, M. N.; Veyrune, J. L. \& Peyron, M. A. The masticatory normative indicator. J. Dent. Res., 89(3):281-5, 2010.

Zembic, A.; Glauser, R.; Khraisat, A. \& Hämmerle, C. H. Immediate vs. early loading of dental implants: 3-year results of a randomized controlled clinical trial. Clin. Oral Implants Res., 21(5):481-9, 2010
Dirección para correspondencia:

Dr. Emanuel Figueroa Ahumada

Departamento Odontológico Área de Salud

Corporación Municipal Viña del Mar

Universidad de Valparaíso

Valparaíso

CHILE

Email: emanuelfiguero@hotmail.com

Recibido : 18-02-2018

Aceptado: 01-06-2018 